\title{
Mass spectrometry of planetary exospheres at high relative velocity: direct comparison of open- and closed-source measurements
}

\author{
Stefan Meyer ${ }^{1, *}$, Marek Tulej ${ }^{1}$, and Peter Wurz ${ }^{1}$ \\ ${ }^{1}$ Physics Institute, Space Research and Planetary Sciences, University of Bern, Sidlerstrasse 5, 3012 Bern, Switzerland \\ * Invited contribution by Stefan Meyer, recipient of the EGU Outstanding Student Poster and PICO Award 2016. \\ Correspondence to: Stefan Meyer (stefan.meyer@space.unibe.ch)
}

Received: 4 August 2016 - Published in Geosci. Instrum. Method. Data Syst. Discuss.: 29 August 2016

Revised: 9 December 2016 - Accepted: 21 December 2016 - Published: 10 January 2017

\begin{abstract}
The exploration of habitable environments on or inside icy moons around the gas giants in the solar system is of major interest in upcoming planetary missions. Exactly this theme is addressed by the JUpiter ICy moons Explorer (JUICE) mission of ESA, which will characterise Ganymede, Europa and Callisto as planetary objects and potential habitats.

We developed a prototype of the Neutral Gas and Ion Mass spectrometer (NIM) of the Particle Environment Package (PEP) for the JUICE mission intended for composition measurements of neutral gas and thermal plasma. NIM/PEP will be used to measure the chemical composition of the exospheres of the icy Jovian moons. Besides direct ion measurement, the NIM instrument is able to measure the inflowing neutral gas in two different modes: in neutral mode, where the gas enters directly the ion source (open source), and in thermal mode, where the gas gets thermally accommodated to the wall temperature by several collisions inside an equilibrium sphere, called antechamber, before entering the ion source (closed source).

We performed measurements with the prototype NIM using a neutral gas beam of 1 up to $4.5 \mathrm{~km} \mathrm{~s}^{-1}$ velocity in the neutral and thermal mode. The current trajectory of JUICE foresees a flyby velocity of $4 \mathrm{~km} \mathrm{~s}^{-1}$ at Europa; other flybys are in the range of 1 up to $7 \mathrm{~km} \mathrm{~s}^{-1}$ and orbital velocity in Ganymede orbits is around $2 \mathrm{~km} \mathrm{~s}^{-1}$. Different species are used for the gas beam, such as noble gases $\mathrm{Ne}, \mathrm{Ar}, \mathrm{Kr}$ as well as molecules like $\mathrm{H}_{2}$, methane, ethane, propane and more complex ones.

The NIM prototype was successfully tested under realistic JUICE mission conditions. In addition, we find that the antechamber (closed source) behaves as expected with pre-
\end{abstract}

dictable density enhancement over the specified mass range and within the JUICE mission phase velocities. Furthermore, with the open source and the closed source we measure almost the same composition for noble gases, as well as for molecules, indicating no additional fragmentation of the species recorded with the antechamber for the investigated parameter range.

\section{Introduction}

JUpiter ICy moons Explorer (JUICE) is an L-class mission of ESA, which will investigate and characterise Ganymede, Europa and Callisto as planetary objects and potential habitats (JUICE Team, 2012; Grasset et al., 2013). The current trajectory of the JUICE spacecraft foresees a flyby velocity of $4 \mathrm{~km} \mathrm{~s}^{-1}$ at Europa, other flybys in the range of 1 up to $7 \mathrm{~km} \mathrm{~s}^{-1}$ and orbital velocity in Ganymede orbits around $2 \mathrm{~km} \mathrm{~s}^{-1}$.

The Particle Environment Package (PEP) carried by JUICE combines remote global imaging with in situ measurements to study the atmospheres, plasma environments, and magnetospheric interactions and to determine global surface composition and chemistry, especially as related to habitability (Barabash et al., 2013).

NIM, the Neutral Gas and Ion Mass spectrometer, is part of the PEP suite and will be used to measure the chemical composition of the regular atmosphere produced by sublimation, energetic particle bombardment and photon interaction with the surface of the icy Jovian moons (e.g. Wurz et al., 2014; Vorburger et al., 2015). The NIM measurements include volatile species, contributions from non-ice material 


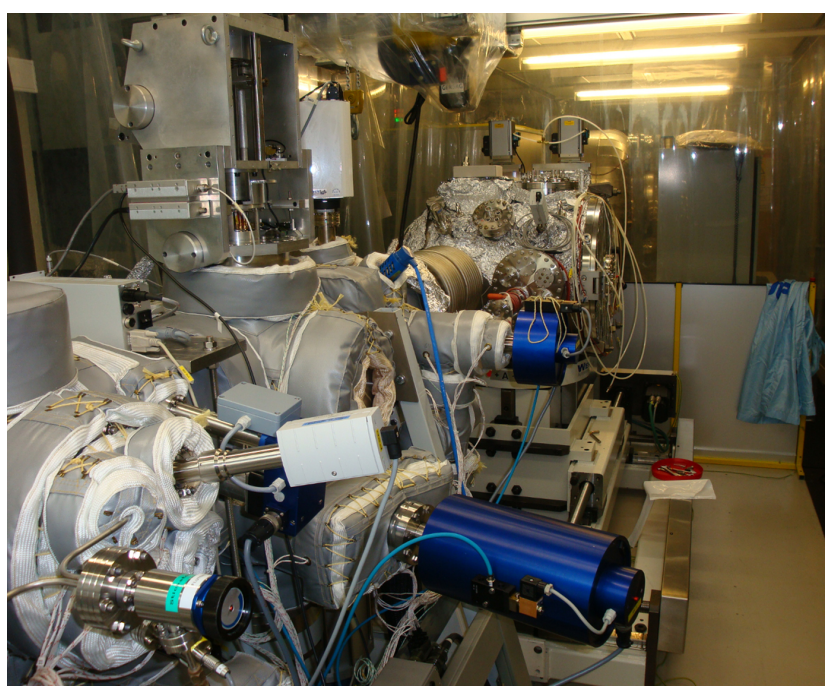

Figure 1. Photograph of CASYMIR calibration facility in clean room area (class 100) with attached UHV chamber for the NIM prototype in the rear.

on the surface and the isotopic composition of major species. In addition, the ion composition of the ionospheres will be measured by direct ion measurement of NIM (ion mode).

We developed a prototype of the NIM instrument, part of PEP, for the JUICE mission and performed measurements with the prototype instrument using a neutral gas beam of 1 up to $4.5 \mathrm{~km} \mathrm{~s}^{-1}$ velocity, containing different species in the neutral (open-source) and thermal (closed-source) mode. The reason for inventing the thermal mode is the large field of view for the neutral gas, which covers basically a half-sphere opening angle. This allows measurement not only near closest approach of a flyby but also at farther distances where the ram direction is outside the narrow field of view of the neutral mode. Furthermore, thermalised neutrals possess low energy spread, which results in best possible performance in terms of mass resolution and transmission. In contrast, the operation in thermal mode may cause complex interactions of the incoming species with the inner wall material of the sphere resulting in fragmentation and chemical alteration. Therefore, the results of these measurements with respect to fragmentation and density enhancements in the closed-source mode are presented here. Furthermore, we give a direct comparison between open- and closed-source mode measurements.

\section{Experimental methods}

\subsection{Calibration facility}

To obtain a neutral gas beam at reasonable velocities, the CASYMIR (calibration system for the mass spectrometer instrument ROSINA) calibration facility has been used in combination with the UHV chamber, housing the NIM prototype.

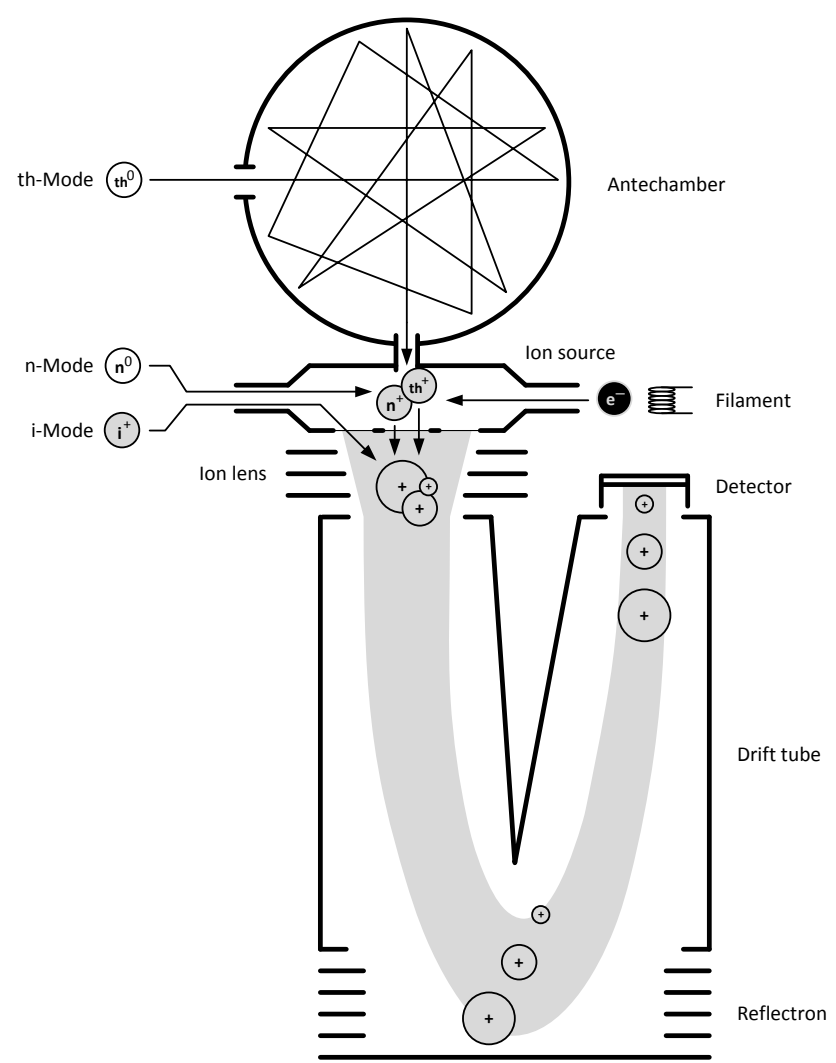

Figure 2. Scheme of NIM prototype illustrating its three operational modes.

The scope of the CASYMIR project was the development of a calibration chamber with the aim of testing and calibrating the two ROSINA mass spectrometers for the Rosetta mission (Graf et al., 2004). CASYMIR consists of a vacuum system with several pumping stages leading to an ultra-high vacuum of a few $10^{-10}$ mbar, where the instrument is attached. A supersonic molecular beam is created by thermal expansion from a heated nozzle up to a maximum speed of $4.5 \mathrm{~km} \mathrm{~s}^{-1}$, which is reached with $\mathrm{H}_{2}$, the lightest gas. The gas mixture for the beam is supplied from a special designed gas mixing unit (GMU) that allows mixtures of different gases. A photograph of the CASYMIR calibration facility with attached UHV chamber in clean room is displayed in Fig. 1. All tests in neutral mode and thermal mode were performed with the NIM prototype installed in the UHV chamber attached to the CASYMIR facility.

\subsection{NIM prototype}

NIM is a time-of-flight instrument with heritage from the RTOF sensor of the ROSINA instrument on the Rosetta mission (Scherer et al., 2006; Balsiger et al., 2007) and the PBACE instrument (Abplanalp et al., 2009). The NIM is designed to operate in three different modes: 


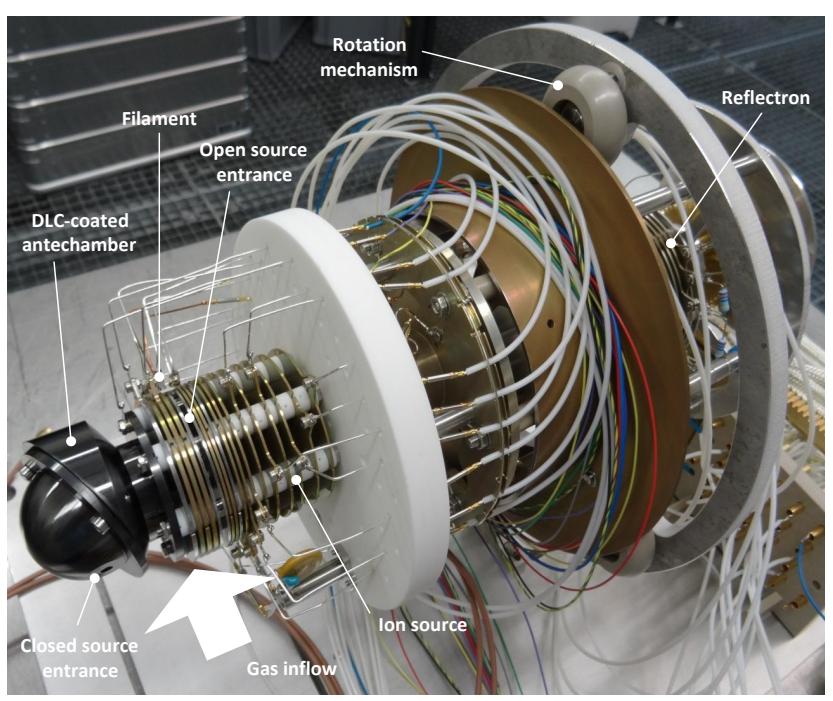

Figure 3. Photograph of NIM prototype with cabling and rotation mechanism.

- In the thermal mode (th-mode), the neutral gas is decelerated from spacecraft velocity down to thermal energies by an equilibrium sphere, called antechamber, and passed on into the ion source in thermal state $\left(\mathrm{th}^{0}\right.$ species in Fig. 2), to be ionised by an electron beam and stored until the subsequent guidance through the ion optics to the detector. This mode (also referred to as closed source in the literature) is used for neutral gas measurements at any mission phase, mainly during Europa torus crossing and all other flybys.

- In the neutral mode (n-mode), the neutral gas $\left(\mathrm{n}^{0}\right.$ species in Fig. 2) enters directly the ion source with the speed relative to the spacecraft, is ionised by an electron beam and subsequently guided through ion optics to the detector. This mode (also referred to as open source in the literature) is used for neutral gas measurements close to the moon, mainly during closest approach at flyby or in orbit phase.

- In the ion mode (i-mode), thermal ions from the ambient plasma $\left(\mathrm{i}^{+}\right.$species in Fig. 2) enter the ion source with the speed relative to the spacecraft and are directly guided through ion optics to the detector. This mode (also referred to as open source in the literature) is used for thermal ion measurements of ionospheric ions close to the moon, mainly in orbit phase or near closest approach during flyby.

These three different modes are illustrated in Fig. 2 together with the entire ion-optical scheme of the NIM prototype instrument. In ion mode (i-mode) the ions from the ambient plasma enter directly into the ion source with spacecraft velocity in ram direction. In neutral mode (n-mode) the neutral

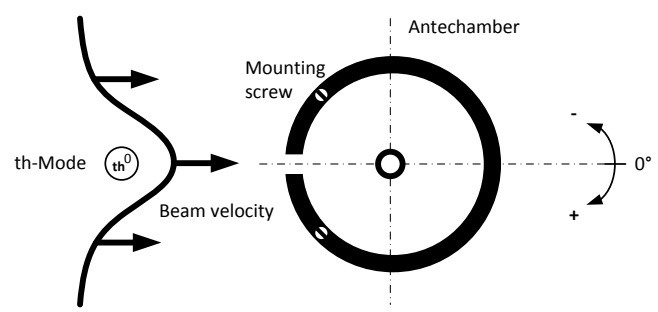

Figure 4. Scheme of neutral gas beam entry in th-mode at CASYMIR measurements. View from top; see Fig. 2.

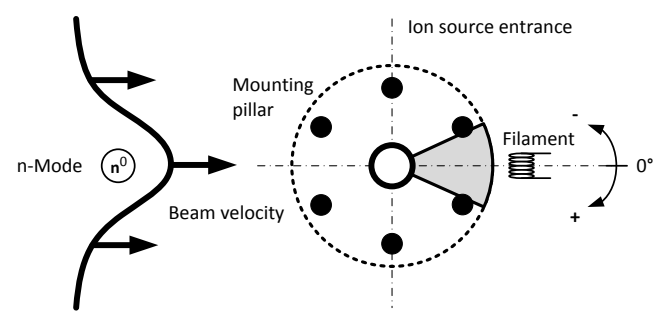

Figure 5. Scheme of neutral gas beam entry in n-mode at CASYMIR measurements. View from top; see Fig. 2.

gas is also entering directly into the ion source with spacecraft velocity in ram direction, which is a so-called open source. The neutral gas is then ionised around the central axis of the ion source by an electron beam produced by a filament and the generated ions are deflected by ion optics towards ion source exit. In thermal mode (th-mode) the neutral gas is first entering a sphere and then decelerated by hitting the interior wall many times, thus establishing thermal equilibrium of the gas with the walls of the antechamber. This is exactly the principle of an antechamber, which is a so-called closed source. The thermalised neutral gas from the antechamber enters the ion source, where it is ionised by the electron beam and the generated ions are again deflected by ion optics towards ion source exit.

The advantage of operation in th-mode is the large field of view for the neutral gas entering a small hole in the antechamber, which covers basically a half-sphere opening angle. This allows measurement not only near closest approach of a flyby but also at farther distances where the ram direction is outside the narrow field of view of the neutral mode. Furthermore, thermalised neutrals do not possess large energy spread (constant energy instead of constant velocity), which results in best possible performance in terms of mass resolution and transmission. The disadvantage of operation in th-mode is the complex interactions of the incoming species with the inner wall material of the sphere resulting in fragmentation and chemical alteration. However, the measurements obtained in th-mode can then be compared with those obtained by the n-mode at closest approach of a flyby or in orbit phase. Furthermore, inter-calibrations between th-mode and $\mathrm{n}$-mode can be done in the laboratory. 
(a)
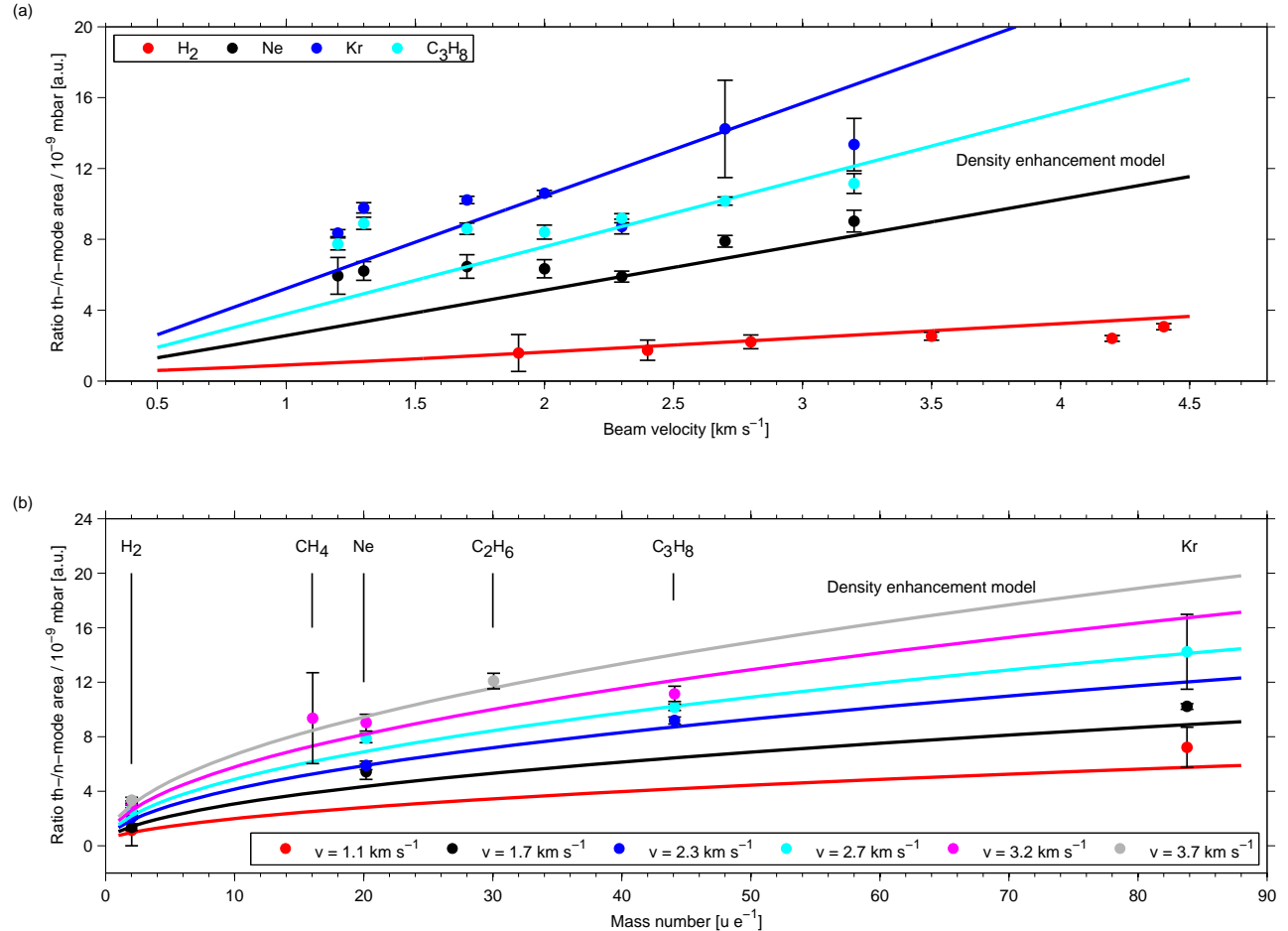

Figure 6. Ratio of signal in thermal mode to signal in neutral mode as function of beam velocity in (a) and as function of mass in (b).

Figure 3 shows a photograph of the NIM prototype with cabling and rotation mechanism, which covers a measurement range of $180^{\circ}\left( \pm 90^{\circ}\right.$ with respect to ram direction and antechamber entrance hole). On the left-hand side of Fig. 3, the tested antechamber with DLC coating can be seen. DLC stands for diamond-like carbon, which is an amorphous carbon material that displays some of the typical properties of diamond. A gold-coated antechamber, which is not depicted here, was tested as well. As has already been discussed above, the antechamber is used in th-mode as closed source to equilibrate the incoming neutral gas beam to thermal energies. The field of view covers basically a half-sphere, in which the effective particle number density is modulated by a cosine of the entrance angle with respect to the normal of the opening hole.

Because of the thermalisation of the incoming gas, the antechamber produces a density enhancement compared to the open source, which can be calculated as follows (Wurz et al., 2007):

$$
\begin{aligned}
& \frac{n_{\mathrm{cs}}}{n_{\mathrm{os}}}=\sqrt{\frac{T_{\mathrm{a}}}{T_{\mathrm{s}}}} \cdot \frac{F(S) \cdot k \cdot \sin ^{2}\left(\frac{\omega}{2}\right) \cdot \cos ^{2}\left(\frac{\omega}{2}\right)}{1-k \cdot \cos ^{2}\left(\frac{\omega}{2}\right)} \cdot \frac{d_{i}^{2}}{d_{i}^{2}+d_{s}^{2}} \\
& F(S)=e^{-S^{2}}+\pi^{\frac{1}{2}} \cdot S \cdot(1+\operatorname{erf}(S)) \\
& S=v_{\mathrm{sc}} \cdot \cos \chi \cdot \sqrt{\frac{m}{2 k_{\mathrm{B}} T_{\mathrm{a}}}},
\end{aligned}
$$

where $n_{\mathrm{cs}}$ is the closed-source number density, $n_{\mathrm{os}}$ is the open-source number density, $T_{\mathrm{a}}$ the ambient gas temperature,
$T_{\mathrm{S}}$ the antechamber temperature, $\omega$ is the cone half-angle of the open source, $v_{\mathrm{sc}}$ the spacecraft velocity and $\chi$ its angle with respect to the surface normal of the entrance aperture, $m$ the mass of the gas, $k$ the probability of a molecule being re-emitted after colliding with the interior surface, $d_{i}$ the opening hole diameter and $d_{s}$ the diameter of the exit hole to the ion source.

\subsection{Measurement method}

Measurements with the NIM prototype, built into the UHV chamber, using a neutral gas beam of realistic velocities in the n-mode (open source) and th-mode (closed source) have been performed. To obtain a neutral gas beam at realistic velocities, the CASYMIR facility has been used (see Sect. 2.1). Different species and gas mixtures are used, such as noble gases $\mathrm{Ne}, \mathrm{Ar}, \mathrm{Kr}$ as well as molecules like $\mathrm{H}_{2}, \mathrm{CH}_{4}, \mathrm{C}_{2} \mathrm{H}_{6}$, $\mathrm{C}_{3} \mathrm{H}_{8}$ and more complex ones.

The used measurement characteristics are discussed in the following. On the one hand, Fig. 4 shows the scheme of neutral gas beam entry in th-mode at the CASYMIR measurements, where the neutral gas beam with Gaussian beam profile impinges on the antechamber opening hole with $4 \mathrm{~mm}$ diameter, which can be rotated $\pm 90^{\circ}$. The antechamber measures $40 \mathrm{~mm}$ in diameter and is DLC-coated inside (goldcoated antechamber is also tested) with mounting screws at $\pm 45^{\circ}$ from $0^{\circ}$ position. 

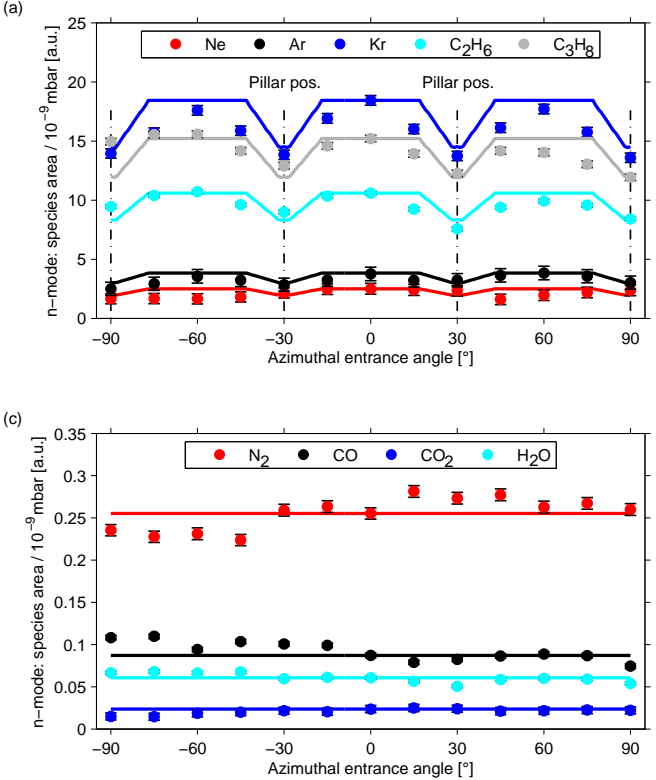

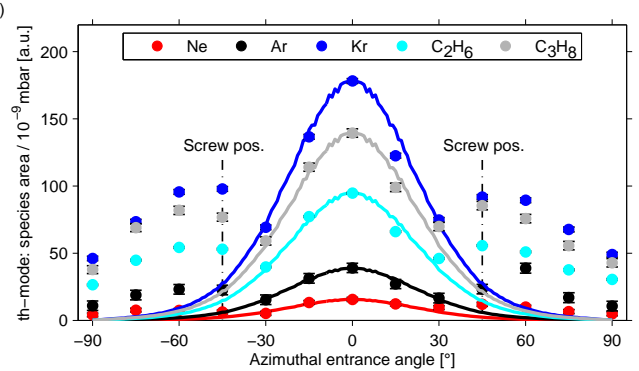

(d)

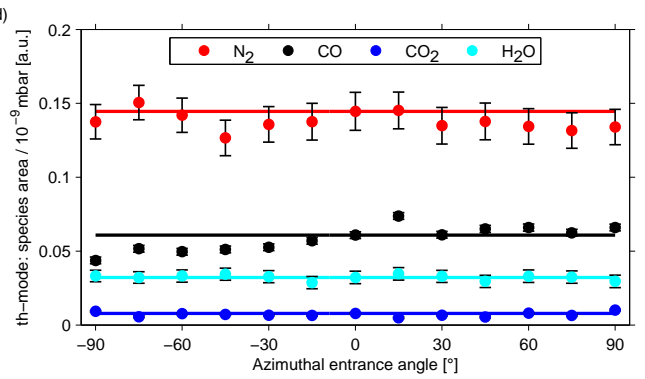

Figure 7. The results of azimuthal rotation campaign conducted in CASYMIR facility. Panels $(\mathbf{a}, \mathbf{b})$ are for species in the neutral beam; panels $(\mathbf{c}, \mathbf{d})$ are for species of the residual gas. Panels $(\mathbf{a}, \mathbf{c})$ are for n-mode measurements; panels $(\mathbf{b}, \mathbf{d})$ are for th-mode measurements.

On the other hand, Fig. 5 shows the scheme of neutral gas beam entry in $n$-mode for the CASYMIR measurements, where the neutral gas beam with Gaussian profile enters the open (line-of-sight) entrance rings, which can be rotated $\pm 90^{\circ}$. The opening angle with respect to elevation is $\sim 11^{\circ}$, which is given by the line-of-sight geometry for the entrance with dimensions $2 \mathrm{~mm} \times 6 \mathrm{~mm}$ (thickness $\times$ width) and additional mounting pillars of $4 \mathrm{~mm}$ every $30^{\circ}$.

Generally, the Gaussian gas beam, with width of about $11 \mathrm{~mm}$ at instrument entrance, has a flux of about $2 \times 10^{12} \mathrm{~cm}^{-2} \mathrm{~s}^{-1}$, which corresponds to a pressure of $10^{-9} \mathrm{mbar}$ of a selected gas mixture at a pressure of about $10^{-10}$ mbar of residual gas in the UHV chamber, mainly consisting of $\mathrm{N}_{2}, \mathrm{H}_{2} \mathrm{O}, \mathrm{CO}$ and $\mathrm{CO}_{2}$ gas. Furthermore, the ionising electron beam was kept at $100 \mu \mathrm{A}$ for all measurements, but can be operated between 10 and $1000 \mu \mathrm{A}$. In fact, all analyses were made based on a final mass spectrum consisting of 100000 accumulated single spectra, which is called one measurement in our case.

\section{Results and discussion}

\subsection{Beam velocity results}

For the beam velocity campaign, measurements with all stated gas mixtures at different velocities ( 1 up to $4.5 \mathrm{~km} \mathrm{~s}^{-1}$ ) for both modes, n-mode and th-mode, at $0^{\circ}$ position each, have been performed. The result summary of this campaign is presented in Fig. 6, where the ratio of the measured peak area normalised to $10^{-9}$ mbar between th-mode and n-mode is shown. In the upper panel a of Fig. 6, the ratio of peak areas of the th-mode to the n-mode, the $\mathrm{c}-\mathrm{o}$ ratio, is plotted versus the beam velocity for the different species in the measured gas mixtures. Additionally, the density enhancement model, described above (see Eq. 1), is given as solid lines for the corresponding molecular masses of the analysed species. In the lower panel b of Fig. 6, the $\mathrm{c}-\mathrm{O}$ ratio is plotted versus the species mass for different beam velocities. Likewise, the density enhancement model, described above (see Eq. 1), is given as solid lines for the corresponding beam velocities.

The measured $\mathrm{c}-\mathrm{O}$ ratio is in good agreement with the calculated density enhancement for noble gases like $\mathrm{Ne}$ and $\mathrm{Kr}$, as well as molecules like $\mathrm{CH}_{4}, \mathrm{C}_{2} \mathrm{H}_{6}$ and $\mathrm{C}_{3} \mathrm{H}_{8}$. Because of the good agreement, it can be extrapolated for all JUICE mission phases. Therefore, the behaviour of the antechamber (closed source) together with the NIM prototype could successfully be verified for gas beam velocities of 1 up to $4.5 \mathrm{~km} \mathrm{~s}^{-1}$, which covers the majority of JUICE mission phases, including Europa flyby and Ganymede orbit. The verified mass range in $n$-mode and th-mode goes from $2\left(\mathrm{H}_{2}\right)$ up to $84 \mathrm{u}(\mathrm{Kr})$, which is most relevant for the JUICE mission. However, NIM supports a mass range up to $1300 \mathrm{u} \mathrm{e}^{-1}$ for a $25 \mu$ s measurement.

\subsection{Azimuthal rotation results}

For the azimuthal rotation campaign, measurements of all stated gas mixtures at different azimuthal entrance angles $\left( \pm 90^{\circ}\right.$ from $0^{\circ}$ position) for both modes, n-mode and thmode, at the same velocity for each gas mixture (2 up to $3 \mathrm{~km} \mathrm{~s}^{-1}$ ) have been conducted. The result summary of this campaign is presented in Fig. 7, where the measured species 

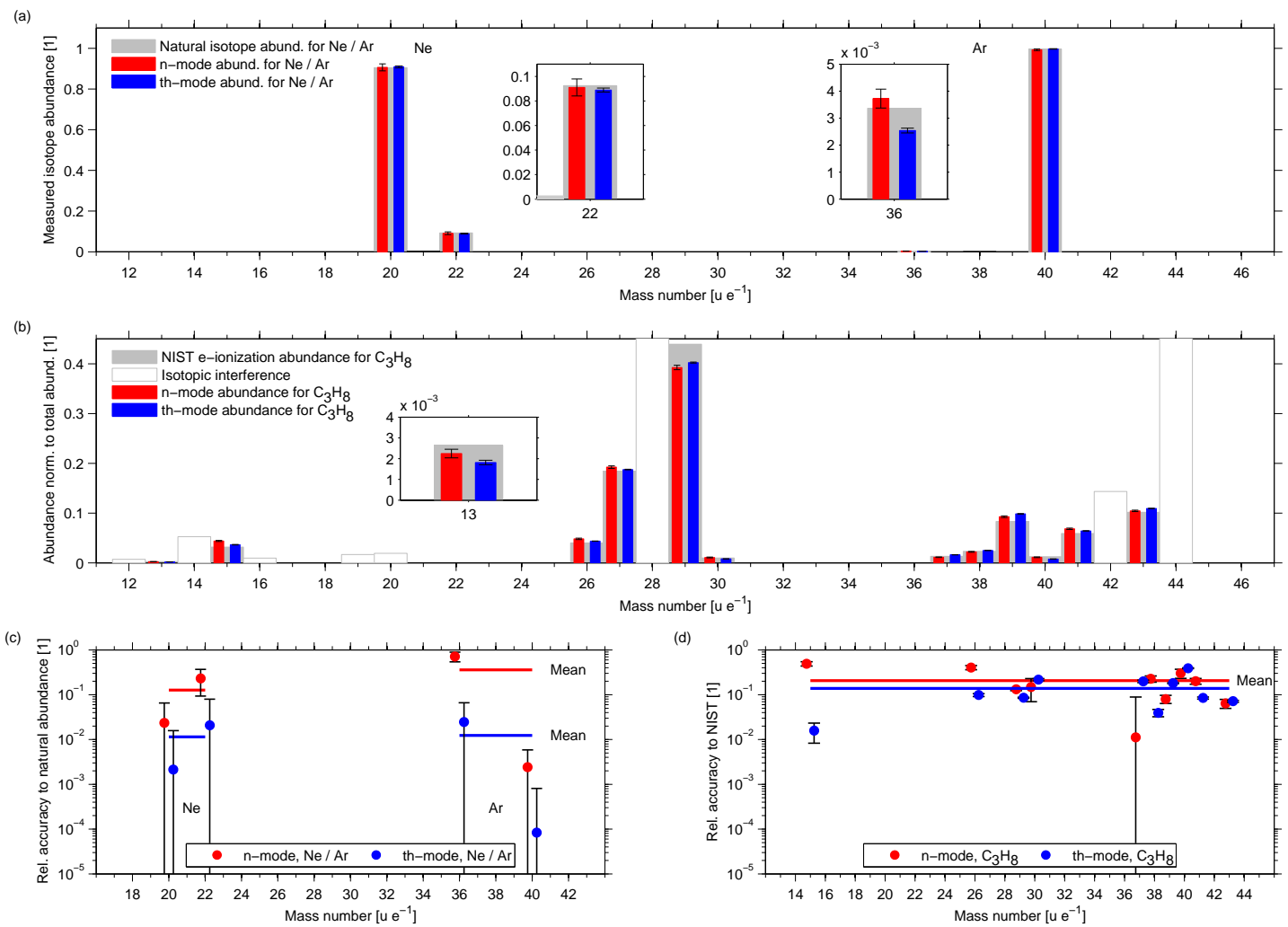

Figure 8. Isotope analysis results of mass spectra from CASYMIR measurements, obtained by summing up 100000 individual spectra.

area normalised to $10^{-9}$ mbar versus azimuthal rotation angle is shown. In the upper left panel a of Fig. 7, the n-mode area is plotted for different species in the measured gas mixtures. The solid lines give the expected behaviour, which is independent (constant) with respect to the azimuthal entrance angle, except for a drop of the signal, where the mounting pillars of the ion source are in line of sight (see Fig. 5). This could be verified with the open-source measurements ranging from -90 to $+90^{\circ}$ for different species in the measured gas mixtures. The beam velocity for this campaign was kept between 2 and $3 \mathrm{~km} \mathrm{~s}^{-1}$ depending on the gas mixture.

In the upper right panel b of Fig. 7, the th-mode area is plotted for different species in the measured gas mixtures. The solid lines give again the expected behaviour, which follows a cosine of the entrance angle modulated by the Gaussian beam profile. In space, the curves are expected to be purely cosine of the angle between entrance aperture of the antechamber and the spacecraft velocity, since the incoming gas is equally distributed in space (at least locally) and has in principle a flat profile. However, the th-mode measurements are in good agreement with the expected profile around $\pm 30^{\circ}$. Outside of $\pm 30^{\circ}$, unexpected side lobes occur with maxima at $\pm 45^{\circ}$, which we attribute to scattering of the gas beam at the mounting screws of the antechamber (see Fig. 4).
The measured species from the residual gas in the vacuum chamber are expected to be independent of the azimuthal rotation angle for both the n-mode and the th-mode, as is shown by the measurement of the residual gas species $\mathrm{N}_{2}, \mathrm{CO}, \mathrm{CO}_{2}$ and $\mathrm{H}_{2} \mathrm{O}$ in n-mode, in the lower left panel $\mathrm{c}$ of Fig. 7, and in th-mode, in the lower right panel d of Fig. 7.

\subsection{Isotope analysis results}

For the isotope analysis, measurements with all stated gas mixtures at velocities between 2 and $3 \mathrm{~km} \mathrm{~s}^{-1}$ for both modes, $\mathrm{n}$-mode and th-mode, at $0^{\circ}$ position each, have been selected. The isotope analysis results for both th-mode and n-mode are shown in Fig. 8. In the top panel a of Fig. 8, the measured isotope abundance is plotted for elements (noble gases like $\mathrm{Ne}$ and $\mathrm{Ar}$ ) in comparison with the natural table of isotopic abundances (CIAAW, 2016). In the bottom left panel c of Fig. 8, its relative accuracy to natural isotopic abundances is given. Here, the measured relative isotopic accuracy to natural abundance reaches from $100 \mathrm{ppm}$ for ${ }^{40} \mathrm{Ar}$ in th-mode up to almost 1 for ${ }^{36} \mathrm{Ar}$ in n-mode, depending on the signal-to-noise ratio (SNR) up to the detection limit. Two additional insets in Fig. 8a show a zoom on the ${ }^{22} \mathrm{Ne}$ isotope and ${ }^{36} \mathrm{Ar}$ isotope, whereas ${ }^{21} \mathrm{Ne}$ and ${ }^{38} \mathrm{Ar}$ are not visible and below the detection limit at this beam flux and NIM operation conditions. The n-mode and th-mode mean accuracies 


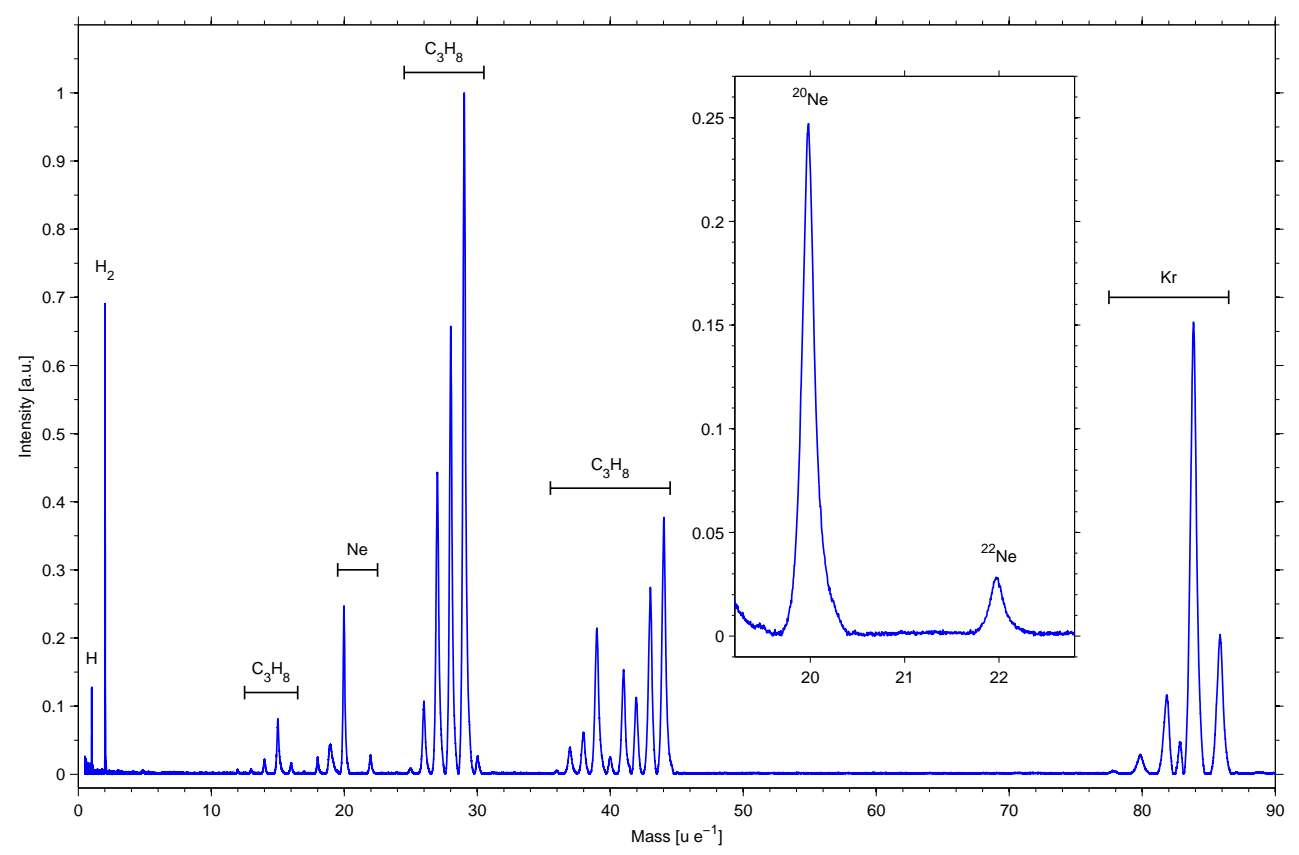

Figure 9. Typical mass spectrum of a th-mode measurement of a gas mixture of $\mathrm{H}_{2}, \mathrm{Ne}, \mathrm{Kr}$ and $\mathrm{C}_{3} \mathrm{H}_{8}$. The spectrum is a background corrected sum of 100000 individual spectra.

are different due to the density enhancement, which also results in a SNR enhancement and therefore a better accuracy for th-mode. In addition, NIM was not optimised for isotope measurements during these campaigns.

In the middle panel $\mathrm{b}$ of Fig. 8, the fragmentation pattern normalised to the total abundance is plotted for the molecule $\mathrm{C}_{3} \mathrm{H}_{8}$ (propane) in comparison with the NIST electron ionisation abundance from NIST Chemistry Webbook (Web NIST, 2016). Mass lines with isotopic interference are marked and omitted for the analysis. An additional inset in Fig. $8 \mathrm{~b}$ shows a zoom on the mass line of $13 \mathrm{u} \mathrm{e}^{-1}$. The measured mean relative isotopic accuracy to NIST electron ionisation abundance, shown in the bottom right panel d of Fig. 8, is between 10 and $20 \%$ for both n-mode and th-mode, which is about the NIST measurement accuracy and suggests, together with the noble gas results, a much higher accuracy for the NIM prototype instrument. Moreover, the direct comparison of the n-mode (open source) and th-mode (closed source) shows the same fragmentation pattern; i.e. there is no evidence for any fractionation effects inside the antechamber, since both modes measure almost the same composition, at least for the measured molecules $\mathrm{H}_{2}, \mathrm{CH}_{4}, \mathrm{C}_{2} \mathrm{H}_{6}$ and $\mathrm{C}_{3} \mathrm{H}_{8}$, as well as methanol vapour and propanol vapour.

A typical mass spectrum, measured in th-mode with a gas mixture of $\mathrm{H}_{2}, \mathrm{Ne}, \mathrm{Kr}$ and $\mathrm{C}_{3} \mathrm{H}_{8}$, is illustrated in Fig. 9. An additional inset in Fig. 9 shows a zoom on the ${ }^{20} \mathrm{Ne}$ and ${ }^{22} \mathrm{Ne}$ isotopes, whereas ${ }^{21} \mathrm{Ne}$ is not visible and below the detection limit at this beam flux and NIM operation conditions.

\section{Conclusion}

The NIM prototype has been successfully tested under realistic JUICE mission conditions. In addition, the antechamber (closed source) behaves as expected with predictable density enhancement over the specified mass range and within the JUICE mission phase velocities. Moreover, n-mode (open source) and th-mode (closed source) measure almost the same composition for noble gases, as well as for molecules, indicating no additional fragmentation of the species inside the antechamber. This holds for both versions of the antechamber, the DLC-coated one and the gold-coated one, respectively, which were both successfully tested.

\section{Data availability}

The data used in this paper can be found in the Supplement.

\section{The Supplement related to this article is available online at doi:10.5194/gi-6-1-2017-supplement.}

Acknowledgements. The authors would like to acknowledge the contribution of a number of people helping in technical preparation of the NIM prototype for the presented investigations, including Stefan Brüngger, Philippe Németh and mechanical workshop, as well as Matthias Lüthi and electronics workshop. The financial support from Swiss National Science Foundation and the PRODEX programme of the Swiss Space Office is acknowledged. 
Edited by: G. Kargl

Reviewed by: J. De Keyser

\section{References}

Abplanalp, D., Wurz, P., Huber, L., Leya, I., Kopp, E., Rohner, U., Wieser, M., Kalla, L., and Barabash, S.: A neutral gas mass spectrometer to measure the chemical composition of the stratosphere, Adv. Space Res., 44, 870-878, doi:10.1016/j.asr.2009.06.016, 2009.

Balsiger, H., Altwegg, K., Bochsler, P., Eberhardt, P., Fischer, J., Graf, S., Jäckel, A., Kopp, E., Langer, U., Mildner, M., Müller, J., Riesen, T., Rubin, M., Scherer, S., Wurz, P., Wüthrich, S., Arijs, E., Delanoye, S., De Keyser, J., Neefs, E., Nevejans, D., Rème, H., Aoustin, C., Mazelle, C., Médale, J.-L., Sauvaud, J. A., Berthelier, J.-J., Bertaux, J.-L., Duvet, L., Illiano, J.-M., Fuselier, S. A., Ghielmetti, A. G., Magoncelli, T., Shelley, E. G., Korth, A., Heerlein, K., Lauche, H., Livi, S., Loose, A., Mall, U., Wilken, B., Gliem, F., Fiethe, B., Gombosi, T. I., Block, B., Carignan, G. R., Fisk, L. A., Waite, J. H., Young, D. T., and Wollnik, H.: ROSINA - Rosetta Orbiter Spectrometer for Ion and Neutral Analysis, Space Sci. Rev., 128, 745-801, doi:10.1007/s11214006-8335-3, 2007.

Barabash, S., Wurz, P., Brandt, P., Wieser, M., Holmström, M., Futaana, Y., Stenberg, G., Nilsson, H., Eriksson, A., Tulej, M., Vorburger, A., Thomas, N., Paranicas, C., Mitchell, D. G., Ho, G., Mauk, B. H., Haggerty, D., Westlake, J. H., Fränz, M., Krupp, N., Roussos, E., Kallio, E., Schmidt, W., Szego, K., Szalai, S., Khurana, K., Xianzhe, J., Paty, C., Wimmer-Schweingruber, R. F., Heber, B., Asamura, K., Grande, M., Lammer, H., Zhang, T., McKenna-Lawlor, S., Krimigis, S. M., Sarris, T., and Grodent, D.: Particle Environment Package (PEP), European Planetary Science Congress 2013, 8-13 September, London, UK, Vol. 8, EPSC2013, 2013.

CIAAW: available at: http://www.ciaaw.org/isotopic-abundances. htm, Commission on Isotopic Abundances and Atomic Weights, last access: 2 February 2016.
Graf, S., Altwegg, K., Balsiger, H., Jäckel, A., Kopp, E., Langer, U., Luithardt, W., Westermann, C., and Wurz, P.: A cometary neutral gas simulator for gas dynamic sensor and mass spectrometer calibration, J. Geophys. Res., 109, E07S08, doi:10.1029/2003JE002188, 2004.

Grasset, O., Dougherty, M. K., Coustenis, A., Bunce, E. J., Erd, C., Titov, D., Blanc, M., Coates, A., Drossart, P., Fletcher, L. N., Hussmann, H., Jaumann, R., Krupp, N., Lebreton, J.P., Prieto-Ballesteros, O., Tortora, P., Tosi, F., and Van Hoolst, T.: JUpiter Icy moons Explorer (JUICE): An ESA mission to orbit Ganymede and to characterise the Jupiter system, Planet. Space Sci., 78, 1-21, doi:10.1016/j.pss.2012.12.002, 2013.

JUICE Team: JUICE assessment study report (Yellow Book), ESA/SRE(2011)18, JUICE Science Study Team, ESA, 2012.

Scherer, S., Altwegg, K., Balsiger, H., Fischer, J., Jäckel, A., Korth, A., Mildner, M., Piazza, D., Rème, H., and Wurz, P.: A novel principle for an ion mirror design in time-offlight mass spectrometry, Int. J. Mass Spectrom., 251, 73-81, doi:10.1016/j.ijms.2006.01.025, 2006.

Vorburger, A., Wurz, P., Lammer, H., Barabash, S., and Mousis, O.: Monte-Carlo Simulation of Callisto's Exosphere, Icarus, 262, 14-29, doi:10.1016/j.icarus.2015.07.035, 2015.

Web NIST: available at: http://webbook.nist.gov/chemistry, National Institute of Standards and Technology, Standard Reference Database, last access: 1 February 2016.

Wurz, P., Balogh, A., Coffey, V., Dichter, B. K., Kasprzak, W. T., Lazarus, A. J., Lennartsson, W., and McFadden, J. P.: Calibration Techniques, Calibration of Particle Instruments, in: Space Physics, edited by: Wüest, M., Evans, D. S., and von Steiger, R., International Space Science Institute, 2007.

Wurz, P., Vorburger, A., Galli, A., Tulej, M., Thomas, N., Alibert, Y., Barabash, S., Wieser, M., and Lammer, H.: Measurement of the Atmospheres of Europa, Ganymede, and Callisto, European Planetary Science Congress 2014, EPSC Abstracts, Vol. 9, ID EPSC2014-504, 2014. 